$A b$ initio calculations in a uniform magnetic field using periodic supercells

W. Cai, G. Galli

November 14, 2003

Physical Review Letters 
This document was prepared as an account of work sponsored by an agency of the United States Government. Neither the United States Government nor the University of California nor any of their employees, makes any warranty, express or implied, or assumes any legal liability or responsibility for the accuracy, completeness, or usefulness of any information, apparatus, product, or process disclosed, or represents that its use would not infringe privately owned rights. Reference herein to any specific commercial product, process, or service by trade name, trademark, manufacturer, or otherwise, does not necessarily constitute or imply its endorsement, recommendation, or favoring by the United States Government or the University of California. The views and opinions of authors expressed herein do not necessarily state or reflect those of the United States Government or the University of California, and shall not be used for advertising or product endorsement purposes. 


\title{
Ab initio calculations in a uniform magnetic field using periodic supercells
}

\author{
Wei Cai and Giulia Galli \\ Lawrence Livermore National Laboratory, University of California, Livermore, CA 94550
}

(Dated: September 18, 2003)

\begin{abstract}
We present a formulation of $a b$ initio electronic structure calculations in a finite magnetic field, which retains the simplicity and efficiency of techniques widely used in first principles molecular dynamics simulations, based on plane-wave basis sets and Fourier transforms. In addition we discuss results obtained with this method for the energy spectrum of interacting electrons in quantum wells, and for the electronic properties of dense fluid deuterium in a uniform magnetic field.
\end{abstract}

PACS numbers: 71.15.-m, 71.10.-w, 71.70.Di

In the last two decades, ab initio electronic structure methods based on density functional theory (DFT) have become mature techniques, which are now widely used to investigate the structural and electronic properties of both condensed and finite systems, e.g. molecules and clusters [1]. In particular, the formulation of $a b$ initio molecular dynamics (MD) [2] has permitted key progress in the prediction of finite temperature properties of materials entirely from first principles. The most widely used implementation of $a b$ initio $\mathrm{MD}$ and of electronic structure calculations for condensed systems is based on pseudopotentials and plane-wave (PW) basis sets. The use of PW has several advantages. The convergence of total energy and force calculations can be controlled by a single parameter (kinetic energy cut-off) and improved to arbitrary accuracy. Atomic forces can be easily computed without evaluating the so-called Pulay contributions [3] and efficient fast Fourier transform (FFT) techniques can be applied. PW basis sets call for the use of periodic boundary conditions (PBC), which conveniently eliminate surface and interface effects and allow for a small simulation cell to mimic the bulk behavior of materials.

To date, most ab initio investigations have focused on ground state properties in the absence of external electromagnetic fields. Due to technical difficulties in describing finite fields within PW formulations and using PBC, almost all studies with electromagnetic fields have been carried out perturbatively. Within this approach, simulations are performed at zero field and electric polarizability and magnetic susceptibility are computed based on linear response theory [4]. While this technique can be used when the applied field is sufficiently small, there are many situations, e.g. condensed systems - notably hydrogen - in stars and planets [5], where the effect of a finite field cannot be treated in a perturbative fashion.

Recently there has been progress in explicitly incorporating a finite electric field in condensed-phase $a b$ initio simulations [6]. A non-peturbative Bloch solution of the Schrödinger's equation in a finite magnetic field was also proposed [7]. Yet no attempt has been made to formulate electronic structure calculations including finite magnetic fields in the context of condensed-phase ab initio MD simulations.
In this paper, we describe a formulation of selfconsistent $a b$ initio calculations within DFT where the effect of a finite, uniform magnetic field is treated in a non-perturbative manner, using algorithms based on PW basis sets and FFT. These algorithms have been key in the development of simple and efficient first principles MD techniques. We present applications of this new method to interacting electrons in a quantum well and dense liquid deuterium in a uniform magnetic field.

- Magnetic periodic boundary conditions. The Hamiltonian of an electron in a periodic potential $V(\vec{r})$ and a uniform magnetic field $\vec{B}$ is

$$
H=\frac{1}{2 m}[\vec{p}+e \vec{A}(\vec{r})]^{2}+V(\vec{r}),
$$

where $\vec{A}(\vec{r})$ is the vector potential $(\vec{B}=\nabla \times \vec{A})$. For uniform $\vec{B}, \vec{A}(\vec{r})$ is not periodic and the electron wave function $\psi(\vec{r})$ cannot satisfy PBC. However, physical observables may still retain translational invariance properties in such conditions. For example, in a classical picture a magnetic field does not do any work when an electron moves from one point to another in space (Lorentz force is always perpendicular to electron velocity), so that the electronic kinetic energy is translationally invariant. This suggests that PBC may be generalized to describe electrons in a uniform magnetic field.

Let $\vec{c}$ be the periodicity of the potential $V(\vec{r})$, i.e., $V(\vec{r}-\vec{c})=V(\vec{r})$. If $\psi(\vec{r})$ is an eigenfunction of $H$, then $\psi(\vec{r}-\vec{c})$ is an eigenfunction of $H^{\prime}$, which differs from $H$ only by its vector potential $\vec{A}^{\prime}(\vec{r})=\vec{A}(\vec{r}-\vec{c})$. When $\vec{B}$ is uniform, $\vec{A}(\vec{r})$ is linear, i.e., $\vec{A}(\vec{r}-\vec{c})=\vec{A}(\vec{r})-\vec{A}(\vec{c})$. Therefore, we can regard the above translation of $H$ as a gauge transformation for $\overrightarrow{A:} \overrightarrow{A^{\prime}}(\vec{r})=\vec{A}(\vec{r})-\nabla \lambda(\vec{r})$, with $\lambda(\vec{r})=\vec{A}(\vec{c}) \cdot \vec{r}$. Gauge invariance insures that $\psi^{\prime}(\vec{r})=\exp \left[i \frac{e}{h} \lambda(\vec{r})\right] \psi(\vec{r})$ is also an eigenfunction of $H^{\prime}$. In the spirit of the Bloch theorem, we can require $\psi(\vec{r}-\vec{c})$ to equal $\psi^{\prime}(\vec{r})$, up to a phase factor $\exp (i \vec{k} \cdot \vec{c})$ :

$$
\psi(\vec{r}-\vec{c})=\exp \left[i \frac{e}{\hbar} \vec{A}(\vec{c}) \cdot \vec{r}-i \vec{k} \cdot \vec{c}\right] \cdot \psi(\vec{r}),
$$

Eq.(2) expresses the so-called magnetic periodic boundary condition (MPBC), which was first suggested in Ref. [8]. 
It is straightforward to see that if the wave function satisfies MPBC, then the charge density $\rho(\vec{r})=|\psi(\vec{r})|^{2}-\mathrm{a}$ measurable quantity, is a periodic function. In the zerofield case, Eq. (2) simply reduces to the Bloch theorem. For simplicity in the following we will only discuss the case of $\vec{k}=0$ ( $\Gamma$ point).

Consider a rectangular simulation cell [for which $V(\vec{r})$ is periodic] of dimension $a$ and $b$ along $x$ and $y$, respectively. Let a uniform magnetic field be parallel to the $z$-axis and adopt the Landau gauge: $\vec{A}(\vec{r})=(0, B x, 0)$. As the wave function is periodic along the $z$-axis, in the following we will not discuss explicitly the $z$-dependence of $\psi$. In the Landau gauge, MPBC can be expressed as,

$$
\begin{aligned}
& \psi(x-a, y)=\exp \left(i \frac{e B a}{\hbar} y\right) \cdot \psi(x, y) \\
& \psi(x, y-b)=\psi(x, y) .
\end{aligned}
$$

An interesting property of MPBC can be obtained by considering the phase change of $\psi$ as one moves along the edge of the simulation cell. One accumulates a total phase of $e B a b / \hbar$ after completing one loop, which must equal $2 n \pi$ ( $n$ integer) for self-consistency. Therefore, the enforcement of MPBC requires the total magnetic flux through the simulation cell to be an integer multiple of the fundamental quanta $\Phi_{0} \equiv h / e: \Phi=B a b=n h / e \equiv$ $n \Phi_{0}$. Depending on the size of the supercell ( $a$ and $b$ ), this quantization imposes a constraint on the magnitude of magnetic fields that one can consider using MPBC.

- Implementing MPBC within a plane-wave-like formulation. We first review the basics of $a b$ initio calculations using PBC and PW basis. In the zero-field case, the real space wave function $\psi(x, y)$ can be expressed by its Fourier components $c\left(k_{x}, k_{y}\right)$, where $k_{x}=n_{x} G_{x}$ and $k_{y}=n_{y} G_{y}, G_{x} \equiv 2 \pi / a, G_{y} \equiv 2 \pi / b\left(n_{x}, n_{y}\right.$ integers $)$. The wave function is then truncated in reciprocal space, $n_{x} \in\left[-N_{x} / 2-1, N_{x} / 2\right], n_{y} \in\left[-N_{y} / 2-1, N_{y} / 2\right]$, so that it is represented by an $N_{x} \times N_{y}$ array of complex numbers. Fast Fourier transform (FFT) techniques can be used to go efficiently from real to reciprocal space representations. Of course the transformation from $\psi(x, y)$ to $c\left(k_{x}, k_{y}\right)$ can be done numerically in one step by a 2-dimensional FFT. However, let us consider a two-step process, where $\psi(x, y)$ is Fourier transformed along the $y$-axis first, leading to $f\left(x, k_{y}\right)$, which is then transformed along the $x$-axis, resulting in $c\left(k_{x}, k_{y}\right)$. We define $f\left(x, k_{y}\right)$ as the wave function in the "intermediate" space, since $k_{y}$ is a reciprocal space variable while $x$ is a real space one. $f\left(x, k_{y}\right)$ can be regarded as a set of one-dimensional periodic functions of $x$, each corresponding to a different $k_{y}$ (there are $N_{y}$ of them in total). Therefore the Fourier transform of $f$ into reciprocal space can be considered as $N_{y}$ individual one-dimensional FFTs along the $x$-axis.

When $B \neq 0, \psi(x, y)$ satisfies MPBC as in Eq. (3). For simplicity consider the smallest field value permitted, $B=h /(e a b)$, i.e., $n=1$. Because $\psi(x, y)$ is periodic in $y$, it can be Fourier transformed into the intermediate space, $\psi(x, y) \stackrel{F F T_{y}}{\longrightarrow} f\left(x, k_{y}\right)$. In this space, MPBC becomes:

$$
f\left(x-a, k_{y}\right)=f\left(x, k_{y}-G_{y}\right) .
$$

Thus the intermediate wave function $f\left(x, k_{y}\right)$ can no longer be regarded as a set of independent, periodic functions of $x$. Instead, all of the $N_{y}$ functions are now interconnected. In fact, if we define a new variable $\hat{x} \equiv x+a k_{y} / G_{y}$, Eq. (4) can be automatically satisfied by letting $f$ be a one-dimensional function of $\hat{x}$,

$$
f(\hat{x}) \equiv f\left(x, k_{y}\right)=f\left(x-a, k_{y}+G_{y}\right)
$$

$f(\hat{x})$ can now be Fourier transformed into the reciprocal space, $f(\hat{x}) \stackrel{F F T_{\hat{x}}}{\longrightarrow} c\left(k_{\hat{x}}\right)$, where the $c\left(k_{\hat{x}}\right)$ are the Fourier coefficients of the wave function into plane-wave-like, orthonormal basis functions satisfying MPBC. The effective reduction of dimensionality (from two to one) of wave functions due to the presence of a magnetic field has been noticed previously [9]. The topology change of the intermediate space is illustrated in Fig. 1 . While $f\left(x, k_{y}\right)$ at $B=0$ can be regarded as a set of independent rings (each representing a periodic function of $x$ for different $k_{y}$ ), it becomes a long spiral when $B=h /(e a b)$. This situation is analogous to that of a crystal lattice containing a screw dislocation [10].

- Evaluation of total energies using $M P B C$. A key step in $a b$ initio simulations is the calculation of $H \psi$, given an arbitrary wave function $\psi$. Once $H \psi$ is computed, iterative algorithms and MD techniques can be applied to compute total energies and forces. When $B=0$, the two components of $H$ - kinetic energy $\hat{T}$ and potential energy $\hat{V}$, are diagonal in reciprocal and real space respectively: $\hat{T} c\left(k_{x}, k_{y}\right)=\frac{\hbar^{2}}{2 m}\left(k_{x}^{2}+k_{y}^{2}\right) c\left(k_{x}, k_{y}\right)$, $\hat{V} \psi(x, y)=V(x, y) \psi(x, y)$. Therefore, $\hat{T} \psi$ and $\hat{V} \psi$ can be easily computed in these two spaces separately, and $\hat{H} \psi$ is obtained by assembling them together via FFT.

When $B=h /(e a b)$, the Hamiltonian can be separated into three components, each diagonal in a different space,

$$
\begin{aligned}
H & =\frac{1}{2 m}[\vec{p}+e \vec{A}(x, y)]^{2}+V(x, y) \\
& =\frac{1}{2 m}\left[\left(-i \hbar \partial_{x}\right)^{2}+\left(-i \hbar \partial_{y}+e B x\right)^{2}\right]+V(x, y) \\
& =-\frac{\hbar^{2}}{2 m} \partial_{x}^{2}+\frac{\hbar^{2} G_{y}^{2}}{2 m a^{2}}\left(x-i \partial_{y} \frac{a}{G_{y}}\right)^{2}+V(x, y) \\
& \equiv \hat{T}_{x}+\hat{T}_{y}+\hat{V}
\end{aligned}
$$

The " $x$-component" of the kinetic energy is diagonal in reciprocal space, $\hat{T}_{x} c\left(k_{\hat{x}}\right)=\hbar k_{\hat{x}}^{2} /(2 m) \cdot c\left(k_{\hat{x}}\right)$. The " $y$ component" becomes a harmonic potential in the intermediate space, $\hat{T}_{y} f(\hat{x})=\hbar^{2} G_{y}^{2} /\left(2 m a^{2}\right) \cdot \hat{x}^{2} f(\hat{x})$. The potential energy is diagonal in real space as usual. Therefore $H \psi$ can be obtained efficiently by calculating these three components separately in three spaces, followed by an assembly via FFT, i.e., 


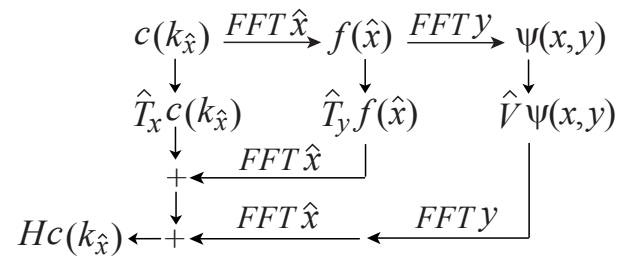

The real- to intermediate- to reciprocal-space Fourier transforms illustrated above apply to wave functions only. DFT calculations also require Fourier transforming the charge density $\rho=|\psi|^{2}$. Since $\rho$ is a simple periodic function in both $x$ and $y$ directions, its Fourier transform can be performed by ordinary 2-dimensional FFT as in the zero-field case.

For simplicity we have only considered the case of the lowest permitted magnetic field. In general, $n=\Phi / \Phi_{0}$ can be larger than 1 . In this case, the intermediate space can be visualized as $n$ spirals interlaced with each other (see Fig. 1). The Fourier transform between intermediate and reciprocal space should then be carried out by $n$ independent one-dimensional FFTs. More technical details will be presented in a forthcoming paper.

- Results. We have implemented the formalism discussed above to study different systems in a magnetic field with increasing levels of complexity. First, as a proof of principle we solved the well known problem of a single electron in a uniform magnetic field and correctly reproduced the energy spectrum of equally spaced Landau levels [11]. We then computed the energy spectrum of a single electron and that of two interacting electrons in a 2-dimensional quantum dot. In Ref. [12], this quantum dot was modeled as a square potential well with energy zero inside and infinite outside the well. Because the electron wave function is entirely localized within the dot, this problem can be solved without using a supercell and plane-wave-like basis functions. As a benchmark for our method, we solve this problem using a supercell enclosing the dot [see inset of Fig. 2(a)]. The potential energy outside the dot is set to $0.1 \mathrm{eV}$. For each magnetic field, we obtain the lowest 64 single electron levels. The energy spectrum of the two-electron system is then obtained by diagonalizing the Hamiltonian matrix in the space spanned by these single electron wave functions. As shown in Fig. 2, the agreement with previous results is very good. The small discrepancy is attributed to the fact that in our study the potential energy outside the quantum dot is not strictly infinite. Our method is readily applicable to the more challenging problem of a periodic array of quantum dots, with electron wave functions not completely localized within each dot. In this case the method of Ref. [12] is no longer applicable.

We also calculated the lowest three levels of a hydrogen atom in magnetic fields [Fig. 3(a)]. Again, supercell techniques are not required for this problem, so that previous data [5] exist for comparison. We used a cubic supercell of $14 \AA$ with an energy cut-off of $10^{3} \mathrm{eV}$, and our results agree very well with earlier reports. The constant difference in the ground state is due to the well known problem for PW to resolve the Coulomb singularity at the nucleus. We also computed the change of binding energy of a hydrogen molecule as a function of magnetic field using the Hartree-Fock approximation [Fig. 3(b)], again in close agreement with earlier results [13]. Notice that the magnetic field here is about seven orders of magnitudes higher than that in Fig. 2. This demonstrates the correctness and accuracy of our method regardless of the magnitude of the magnetic field.

Finally we implemented our formalism within the local density approximation (LDA) of DFT and carried out self-consistent calculations of the electronic properties of dense fluid deuterium [Fig. 4(a)]. The positions of 128 deuterium ions are obtained from a snapshot of an earlier $a b$ initio MD simulation [14] at temperature $5000 \mathrm{~K}$ and density $5 \times 10^{5} \mathrm{~mol} / \mathrm{m}^{3}$ under zero magnetic field. The simulation cell is a cube with length $7.52 \AA$ and an energy cut-off of $2.7 \times 10^{3} \mathrm{eV}$ is used. We have found that the instantaneous band gap $E_{g}$ is strongly influenced by the magnetic field: $E_{g}=0.176 \mathrm{eV}$ at $B=0$ with $E_{g}=0.272 \mathrm{eV}$ at $B=10^{4} \mathrm{~T}$. While no appreciable difference is observed in the total charge density at these two magnetic fields (which is somewhat surprising), the density of individual electronic levels changes dramatically. Fig. 4(b) plots the charge density of the highest occupied molecular orbital (HOMO) for both $B=0$ (blue) and $B=10^{4} \mathrm{~T}$ (red). We see that this state is associated with different atoms at these two magnetic field values. Similar considerations apply to the lowest unoccupied molecular orbital (LUMO). Therefore we expect a strong magnetic field to have a significant influence on the electromagnetic and optical response of compressed fluid deuterium.

In summary, we developed a method for ab initio calculations in the presence of a uniform magnetic field. Our approach retains the simplicity and efficiency of electronic structure calculations based on PW and FFT, and can be applied to both finite and condensed systems. We have also applied the method to quantum wells and compressed deuterium in strong fields, showing the accuracy and efficiency of the method. Calculations of ionic forces and thus the extension of the present method to ab initio MD simulations is expected to be straightforward, at least in the case of local pseudopotentials. The generalization of the approach to include a coupling of the magnetic field with spin degrees of freedom is underway.

We thank E. Pollock for useful discussions and W. Krauss for help on visualization. This work was performed under the auspices of U.S. Department of Energy by University of California Lawrence Livermore National Laboratory under contract No. W-7405-Eng-48. W. C. is supported by the University Relationship Program at LLNL. 
[1] R. M. Martin, Electronic Structure: Basic Theory and Practical Methods, (Cambridge University Press, Cambridge, 2003).

[2] R. Car and M. Parrinello, Phys. Rev. Lett. 55, 2471 (1985)

[3] P. Pulay, in K. P. Lawley ed., Ab Initio Methods in Quantum Chemistry II, (Wiley, Chichester, 1987), p. 241.

[4] S. Baroni, P. Giannozzi and A. Testa, Phys. Rev. Lett. 58, 1861 (1987). F. Mauri and S. G. Louie, ibid. 76, 4246 (1996); F. Mauri, B. Pfrommer and S. G. Louie, ibid. 77, 5300 (1996).

[5] H. Ruder, G. Wunner, H. Herold and F. Geyer, Atoms in Strong Magnetic Fields, (Springer-Verlag, Berlin, 1994).

[6] P. Umari and A. Pasquarello, Phys. Rev. Lett. 89, 157602 (2002).

[7] A. Trellakis, Phys. Rev. Lett. 91, 056405 (2003).

[8] E. Brown, Phys. Rev. 133, A1038 (1964).

[9] G. M. Obermair and H.-J. Schellnhuber, Phys. Rev. B 23, 5185 (1981).

[10] J. P. Hirth and J. Lothe, Theory of Dislocations, (Wiley, New York, 1982).

[11] L. D. Landau and E. M. Lifshitz, Quantum Mechanics, 3rd ed. (Pergamon, Oxford, 1977).

[12] C. E. Creffield, J. H. Jefferson, S. Sarkar, D. L. J. Tipton, Phys. Rev. B 62, 7249 (2000).

[13] T. Detmer, P. Schmelcher, F. K. Diakonos, L. S. Cederbaum, Phys. Rev. A, 56, 1825 (1997).

[14] G. Galli, R. Q. Hood, A. U. Hazi, F. Gygi, Phys. Rev. B, 61, 909 (2000); S. Bonev, B. Militzer and G. Galli, Phys. Rev. B (submitted).

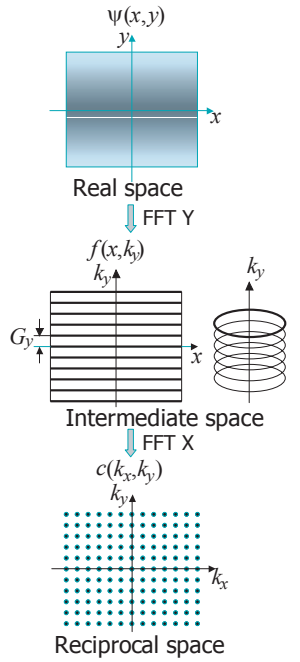

(a)

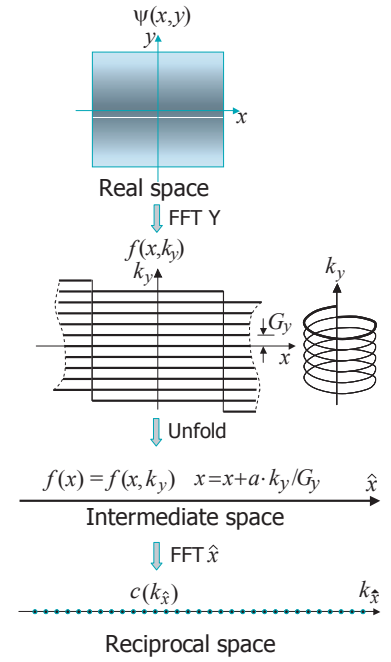

(b)
FIG. 1: The real-space wave function $\psi(x, y)$ can be Fourier transformed into reciprocal-space $c\left(k_{x}, k_{y}\right)$ in two steps, via an intermediate-space wave function $f\left(x, k_{y}\right)$ (see text). (a) At $B=0, f\left(x, k_{y}\right)$ can be regarded as a set of one-dimensional periodic functions, or rings. (b) At $B=h /(e a b)$, MPBC requires $f\left(x, k_{y}\right)$ to be a long spiral. The resulting wave function in intermediate- and reciprocal-space is effectively onedimensional.

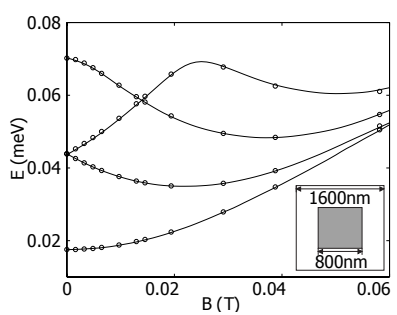

(a)

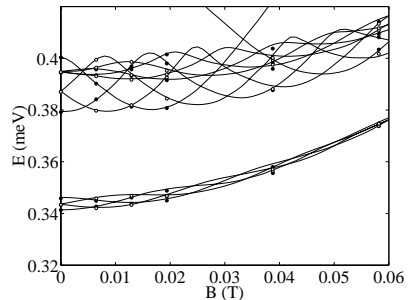

(b)
FIG. 2: (a) Energy spectrum of single electron in quantum dot as a function of magnetic field. The inset shows the geometry of quantum dot (shaded area) and simulation cell (outer square). (b) Energy spectrum of two interacting electrons in quantum dot as a function of magnetic field. Filled and open circles indicate spin singlet and triplet states from this work. Thick and thin lines are for singlet and triplet states from Ref. [12]. 


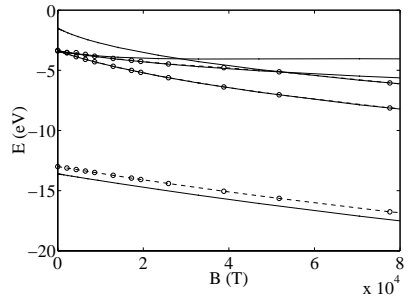

(a)

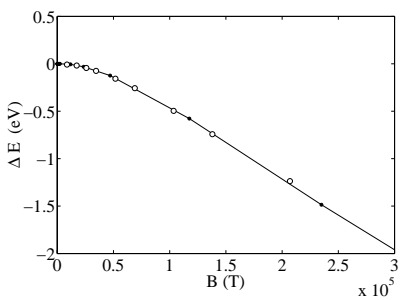

(b)

FIG. 3: (a) Lowest levels of a hydrogen atom as a function of magnetic field: $\circ$ for this work, solid line for previous results [5]. (b) Change of binding energy of an $\mathrm{H}_{2}$ molecule in the ${ }^{1} \Sigma_{g}$ state (two spins antiparallel) as a function of magnetic field: open circles for our result using Hartree-Fock approximation with inter-proton distance fixed at $0.74 \AA$, filled circles and solid line from Ref. [13].

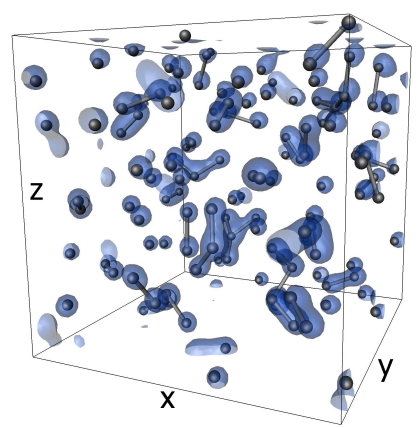

(a)

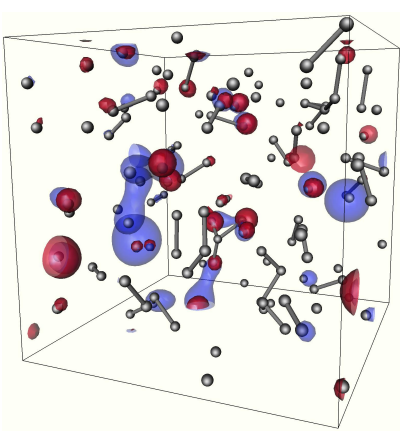

(b)

FIG. 4: (color) (a) Total charge density of a dense deuterium fluid (see text), which remains essentially the same as $B$ goes from 0 to $10^{4} \mathrm{~T}$. (b) The charge densities of HOMO state for $B=0$ (blue) and $B=10^{4} \mathrm{~T}$ (red) are distributed on different atoms. 